\title{
The Current Concepts of Orthodontic Discrepancy Stability
}

\author{
Maen Mahfouz \\ Department of Orthodontics and Pediatric Dentistry, Arab American University, Jenin, Palestine \\ Email: mnmahfouz2005@yahoo.com
}

Received 1 March 2014; revised 4 April 2014; accepted 12 April 2014

Copyright (C) 2014 by author and Scientific Research Publishing Inc. This work is licensed under the Creative Commons Attribution International License (CC BY). http://creativecommons.org/licenses/by/4.0/

(c) (i) Open Access

\begin{abstract}
There is a wide range of opinions on the best time to start orthodontic treatment but the vast majority is carried out on children who have lost all their baby (deciduous) teeth and have most of their adult teeth (except for wisdom teeth) present in the mouth. Stability of orthodontic discrepancy is one of the most important factors that determine when start orthodontic treatment of the dentofacial deformaties. This paper threw light on stability of orthodontic discrepancy, its classification, comparing between stable versus unstable orthodontic discrepancy and its impact on the timing of orthodontic treatment.
\end{abstract}

\section{Keywords}

Stability, Orthodontic, Discrepancy

\section{Introduction}

Orthodontic treatment should be in harmony with modern medical thought: "It is better to prevent than to cure". We should not treat the symptom; we should treat the cause. The therapeutic devices available for this endeavor are not complex, but deciding which ones to use and when to employ them is far from simple. The therapeutic choice is nothing more than the last act in a complete diagnostic process. To be capable of determining the optimum moment to begin treatment, orthodontists must possess a profound comprehension of fundamental information that forms the basis of understanding the pathogenesis of different malocclusions: Normal and pathologic craniofacial growth, orofacial functional behavior, morphogenesis of the dental arches, and child psychology. Armed with this knowledge, orthodontists can accurately discern which discrepancies would benefit from early treatment and not fall into the trap of indiscriminately treating every patient they examine during the mixed dentition stage [1].

If the clinician wants to maximize the benefits of growth and cooperation without subjecting every child to 
four or more years of treatment? From the eruption of the first primary tooth until the development and eruption of the wisdom teeth, the developing dentition should be monitored and interceptive treatment prescribed as necessary. There is a difference, however, between treatment decisions that are thrust upon us due to aberrations of dental development and types of malocclusion that we may choose to treat early by use of appliance therapy or elective extraction of teeth. The management of certain problems such as skeletal discrepancies or crowding can be undertaken at differing times during the dental development [2].

Proffit and Ackerman [3] introduced the concept of the envelope of discrepancy to graphically illustrate how much change can be produced by various types of treatment (Figure 1 and Figure 2). This diagram helps simplify the relationship of the three basic treatment possibilities for skeletal discrepancies. The inner circle, or envelope, represents the limitations of camouflage treatment involving only orthodontics; the middle envelope illustrates the limits of combined orthodontic treatment and growth modification; and outer envelope shows the limits of surgical correction.

Growth modification, generally referred to as dentofacial orthopedics, is the most desirable approach to a severe skeletal problem when the potential for further growth exists. Although the pattern of growth can be favorably modified for some patients, the capacity for major increments in growth is rather limited. The variation in response of individual patients, however, suggests that growth modification should be attempted in preadolescent patients, and parents should be warned that it might not succeed. When a moderate skeletal discrepancy exists and there is no potential for further growth (or if more change is required than can be accomplished through growth modification alone), orthodontic camouflage should be considered. The teeth are repositioned to establish normal overjet and overbite in an effort to compensate for the jaw discrepancy. In a moderate skeletal Class II malocclusion involving mandibular deficiency, the maxillary incisors can be retracted and the mandibular incisors proclined to establish overjet. Extraction of some teeth will usually be required so that enough space in the arch can be created to allow significant movement of other teeth. Such treatment cannot be considered successful if it results in a reasonable dental occlusion at the expense of facial esthetics. In addition, there are limits to how far the periodontium will accommodate the displacement of teeth from their normal positions. Consideration of camouflage requires careful examination for the patient's ultimate facial esthetics and occlusal stability.

The final treatment option for a severe skeletal discrepancy is orthognathic surgery. Once growth has ceased, surgery becomes the only means of correcting a severe jaw discrepancy. Although surgery may allow greater changes, there are still limitations to the surgical options, depending on the type of problem and direction of desired jaw movement, and certain problems are more receptive to surgical correction than others. When dental compensation is present, either naturally or previously produced by orthodontic treatment, these dental positions must be reversed before surgical repositioning of the jaws. The greater the dental compensation is, the smaller the magnitude of jaw movement the surgeon has to correct the skeletal discrepancy. The term reverse orthodontics is often used in reference to the deliberate movement of teeth in a direction that appears to make the occlusion worse initially when preparing the dentition for orthognathic surgery. When dental compensations exist, they limit the distance and the jaws that can be repositioned to achieve a desirable esthetic result. Greater change can be expected when treating a child (who most likely has some remaining potential for growth) with orthodontic tooth movement plus growth modification than for an adult with camouflage orthodontics alone. Consequently, given the same severity of skeletal deformities in both a child and an adult, orthodontics alone in the child may produce a desirable result, whereas the adult would not be manageable without a surgical option. One answer to the question of "When is a problem too severe for orthodontic treatment only?" is "When the combination of tooth movement and growth modification does not have the potential to bring the patient to normal occlusion." In a growing child, a malocclusion that cannot be corrected by orthodontics in addition to growth modification is severe enough that it merits consideration of a surgical plan. In the non-growing patient, if the malocclusion is too severe to be treated with camouflage orthodontic tooth movement, then a surgical treatment plan should be implemented to obtain a reasonable result. The envelope of discrepancy is based on occlusal considerations, and esthetic limits apply. Merely obtaining an ideal occlusion at the expense of compromised facial esthetics does not constitute a successful treatment outcome [4].

This paper is concerned with stability nature and the classifying type of orthodontic discrepancy which is the corner stone for successful communication and illustration for orthodontic patients regarding the sharing decision whether to start treatment now or later.

\subsection{Defining the Stability of Orthodontic Discrepancy}

The stability of orthodontic discrepancy as a term describes the type of orthodontic discrepancy deviation by 


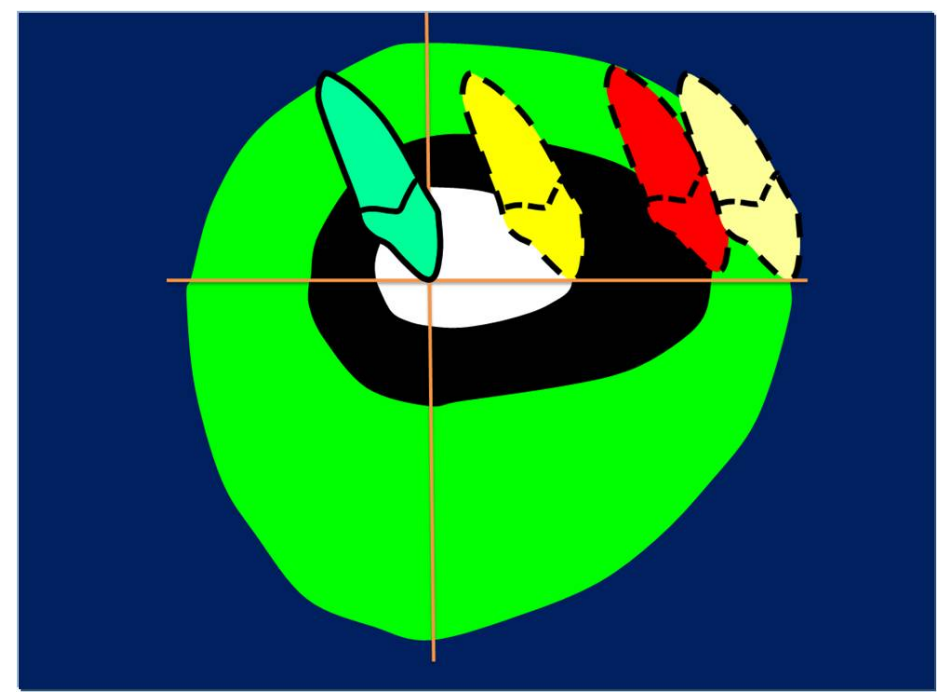

Figure 1. With the ideal position of the upper incisors shown by the origin of the $\mathrm{x}$ and $\mathrm{y}$ axes, the envelope of discrepancy shows the amount of change that could be produced by orthodontic tooth movement alone (the inner envelope of each diagram); orthodontic tooth movement combined with growth modification (the middle envelope) and orthognathic surgery (the outer envelope). Note that the possibilities for each treatment are not symmetric with regard to the planes of space vertical and anteroposterior. There is more potential to retract than procline teeth and more potential for extrusion than intrusion.

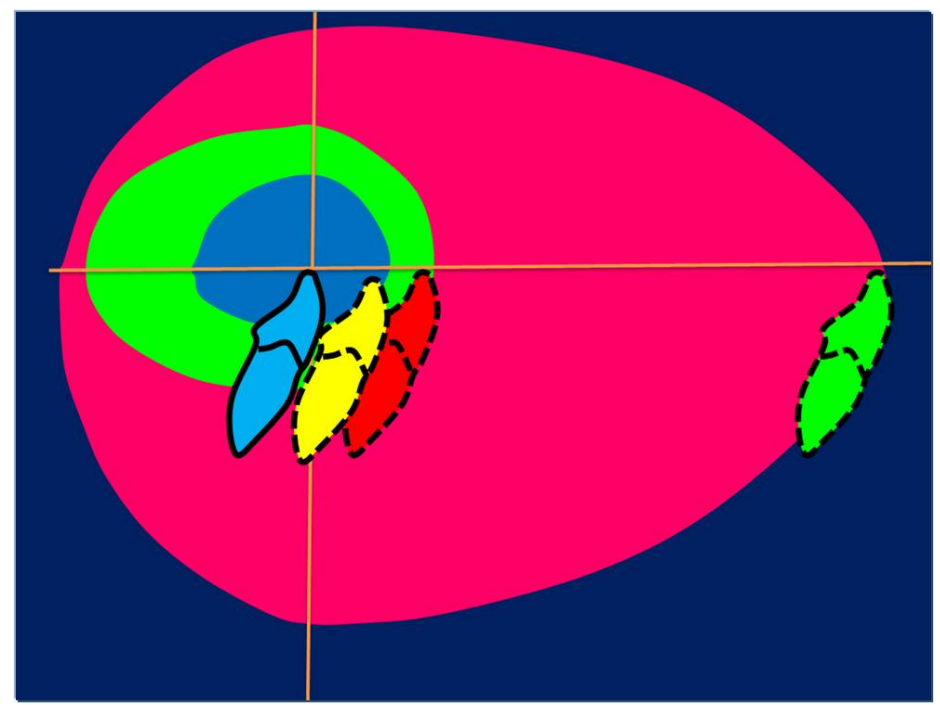

Figure 2. With the ideal position of the lower incisors shown by the origin of the $\mathrm{x}$ and $\mathrm{y}$ axes, the envelope of discrepancy shows the amount of change that could be produced by orthodontic tooth movement alone (the inner envelope of each diagram); orthodontic tooth movement combined with growth modification (the middle envelope) and orthognathic surgery (the outer envelope). Note that the possibilities for each treatment are not symmetric with regard to the planes of space vertical and anteroposterior. There is more potential to retract than procline teeth and more potential for extrusion than intrusion. Since growth of the maxilla cannot be modified independently of the mandible, the growth modification envelope for the two jaws is the same. Surgery to move the lower jaw back has more potential than surgery to advance it. 
time if it increases, decreases or stays as it is. Orthodontic discrepancy has the following features:

1) Quantitative: Describes the magnitude and severity of discrepancy in metric measurements.

2) Qualitative: Describes the quality of discrepancy in classes and types.

3) Dynamics: Describes the stability of discrepancy and its changes by age either soft and/or hard tissue.

Whatever the type of orthodontic discrepancy: Dental, functional and/or skeletal is, the corner stone for successful management of any type of orthodontic discrepancy is through determination its stability nature followed by classifying the type of orthodontic discrepancy. Actually there are two main classes of orthodontic discrepancy depending on its stability, Class I which is stable orthodontic discrepancy while Class II is unstable orthodontic discrepancy, additionally Class II unstable orthodontic discrepancy could be divided into two divisions: Regressive orthodontic discrepancy and Progressive orthodontic discrepancy (Figures 3-5).

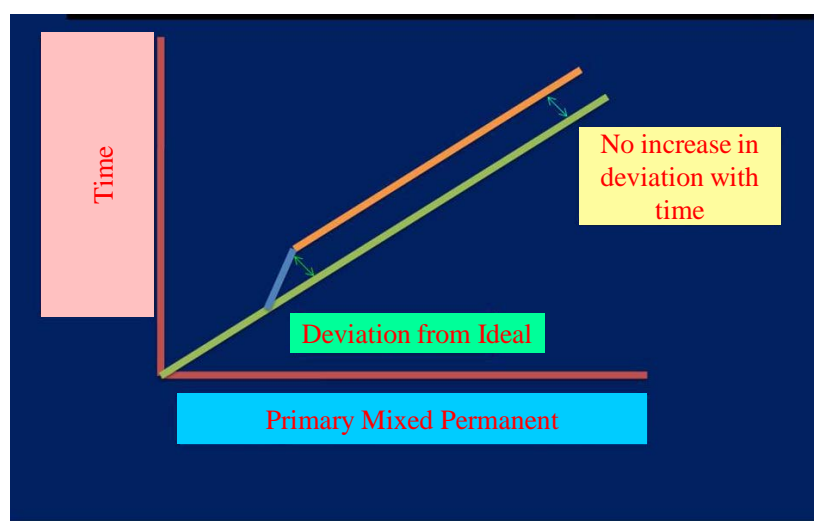

Figure 3. Stable discrepancy.

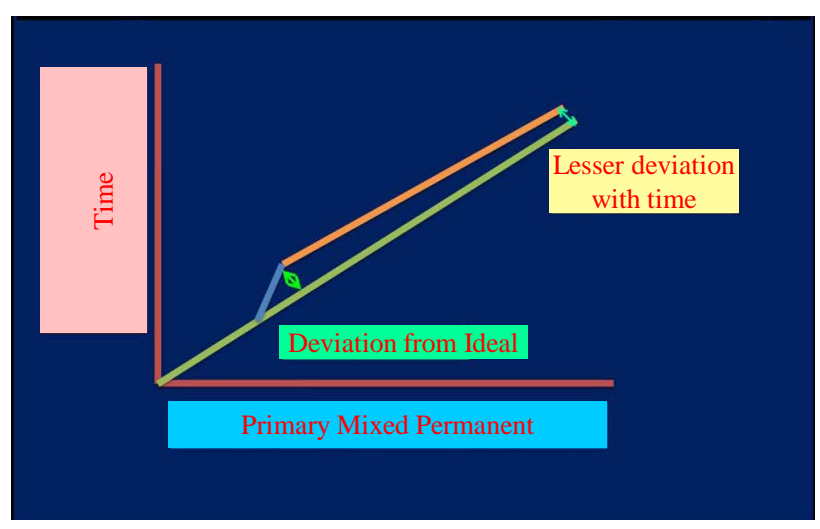

Figure 4. Regressive discrepancy.

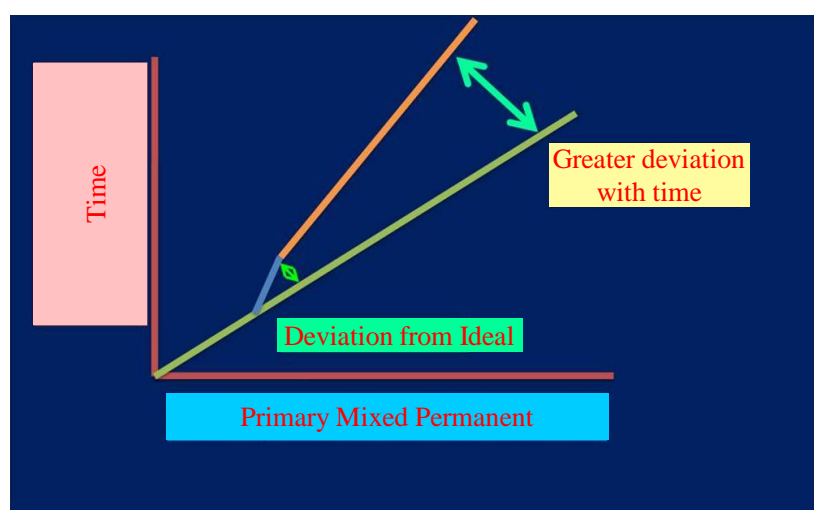

Figure 5. Progressive discrepancy. 
So every orthodontist should ask the following question if he/she is reluctant about when start orthodontic treatment "Is this orthodontic discrepancy is stable or not?" if it is stable orthodontist could postpone treatment and start treatment later unless it is not stable so. Which division is it regressive or progressive? In case of regressive orthodontic discrepancy class, there will be improving in the clinical situation in contrast to the progressive class in which worsening in the clinical situation will be.

It is possible now to be at least semi-quantitative about the limits of orthodontic treatment, in the context of producing normal occlusion. As the diagrams of the "envelope of discrepancy" (Figure 1, Figure 2, Figure 6 and Figure 7) indicate, the limits vary both by the tooth movement that would be needed (teeth can be moved further in some directions than others) and by the patient's age (the limits for tooth movement change little if any with age, but growth modification is possible only while active growth is occurring) [5] [6].

Growth modification in children enables greater changes than are possible by tooth movement alone in adults, as some conditions that could have been treated with orthodontics alone in children (e.g., a centimeter of overjet) become surgical problems in adults. On the other hand, some conditions that initially might look less severe (e.g., 5 $\mathrm{mm}$ of reverse overjet), can be seen even at an early age to require surgery if they are ever to be corrected [5].

For patients whose orthodontic problems are so severe that neither growth modification nor camouflage offers a solution, surgery to realign the jaws or reposition dentoalveolar segments is the only possible treatment option left. One indication for surgery obviously is a malocclusion too severe for orthodontics alone [7].

The envelope of discrepancy (Figure 1, Figure 2, Figure 6 and Figure 7) shows the amount of change that could be produced by orthodontic tooth movement alone (the inner envelope of each diagram); orthodontic tooth movement combined with growth modification (the middle envelope) and orthognathic surgery (the outer envelope). Note that the possibilities for each treatment are not symmetric with regard to the planes of space. There is more potential to retract than procline teeth and more potential for extrusion than intrusion. Since growth of the maxilla cannot be modified independently of the mandible, the growth modification envelope for the two jaws is the same. Surgery to move the lower jaw back has more potential than surgery to advance it [5] [6].

Keep in mind that the envelope of discrepancy outlines the limits of hard tissue change toward ideal occlusion, if other limits due to the major goals of treatment do not apply. In fact, soft tissue limitations not reflected in the envelope of discrepancy often are a major factor in the decision for orthodontic or surgical-orthodontic treatment. Measuring millimeter distances to the ideal condylar position for normal function is problematic, and measuring distances from ideal esthetics is impossible [5].

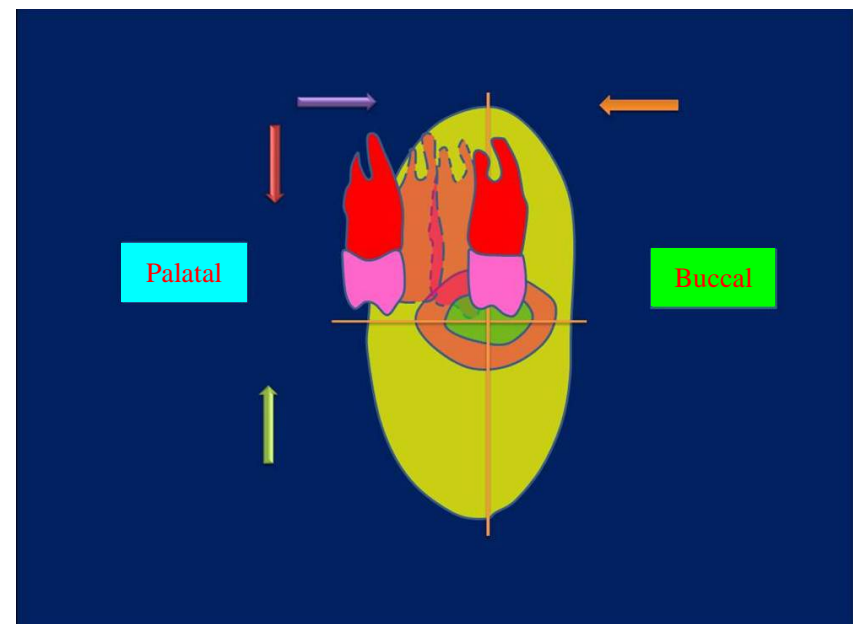

Figure 6. With the ideal position of the upper incisors shown by the origin of the $\mathrm{x}$ and $\mathrm{y}$ axes, the envelope of discrepancy shows the amount of change that could be produced by orthodontic tooth movement alone (the inner envelope of each Diagram); orthodontic tooth movement combined with growth modification (the middle envelope) and orthognathic surgery (the outer envelope). Note that the possibilities for each treatment are not symmetric with regard to the transverse and vertical planes of space. 


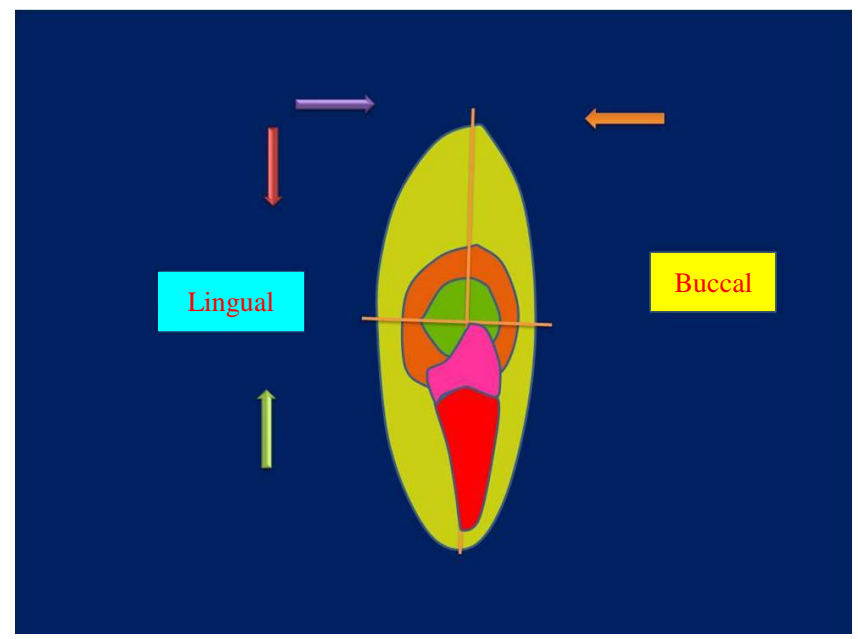

Figure 7. With the ideal position of the lower incisors shown by the origin of the $\mathrm{x}$ and $\mathrm{y}$ axes, the envelope of discrepancy shows the amount of change that could be produced by orthodontic tooth movement alone (the inner envelope of each diagram); orthodontic tooth movement combined with growth modification (the middle envelope) and orthognathic surgery (the outer envelope). Note that the possibilities for each treatment are not symmetric with regard to the transverse and vertical planes of space.

\subsection{Clinical Applications of Stability of Orthodontic Discrepancy}

1) Stable Orthodontic Discrepancy: Spacing between teeth is one of the examples that demonstrates a type of orthodontic discrepancy which is considered stable as it doesn't worsen by time especially if there is good interdigitation with horizontal growth pattern, the similar situation is in the mild displacement of contact points between teeth which is another type of orthodontic discrepancy as it is the least demanded for orthodontic treatment as shown in (Figure 3, Figure 8 and Figure 9).

2) Unstable Orthodontic Discrepancy is either to be regressive orthodontic discrepancy and/or progressive orthodontic discrepancy: Regressive orthodontic discrepancy as in case of ectopic eruption of lower permanent incisors and diastema between central incisors (Figure 4, Figure 5, Figures 10-13), While Progressive orthodontic discrepancy like ectopic eruption of canines or molars and pseudo Class III malocclusion [5] (Figure 4, Figure 5, Figures 14-18).

In case that ectopic eruption of lower permanent incisors is regressive as this ectopic eruption due to over retained primary incisors by time is self corrected without orthodontic intervention by tongue movement. The same matter with the diastema between central incisors in early mixed dentition is self limiting as this space tends to close spontaneously when the canines erupt and the incisor root and crown positions change [5] (Figure 4, Figure 5, Figures 10-13).

In contrast, the ectopic eruption of canines or molars and pseudo Class III malocclusion which is left untreated further deterioration will occur [8] [9].

\section{Discussion}

The stability of orthodontic discrepancy in its broader term is relatively a theoretical concept in spite that it is practical matter as it is simplified the description of orthodontic discrepancy depending on its stability nature as well as facilitate the communication and illustration for orthodontic patients to decide to start treatment early or later as the trend nowadays regarding the decision is a sharing between the orthodontist and patient.

The benefits of orthodontic discrepancy early treatment regarding functional crossbite, maxillary canine eruption, and excessive overjet with a trauma risk have been accepted. Regarding Class II malocclusion, studies have not shown early orthodontic treatment to be more beneficial than treatment started later in adolescence in the permanent dentition. Early Class II treatment has, however, been considered effective in reducing the difficulty of and priority for Phase 2 treatment (King G.J., Wheeler 1999) [10]. 


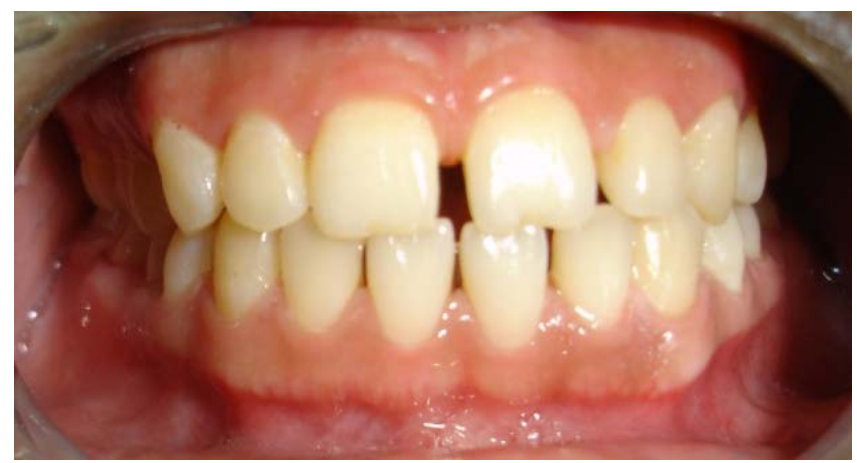

Figure 8. Frontal-intraoral view showing Spacing between teeth.

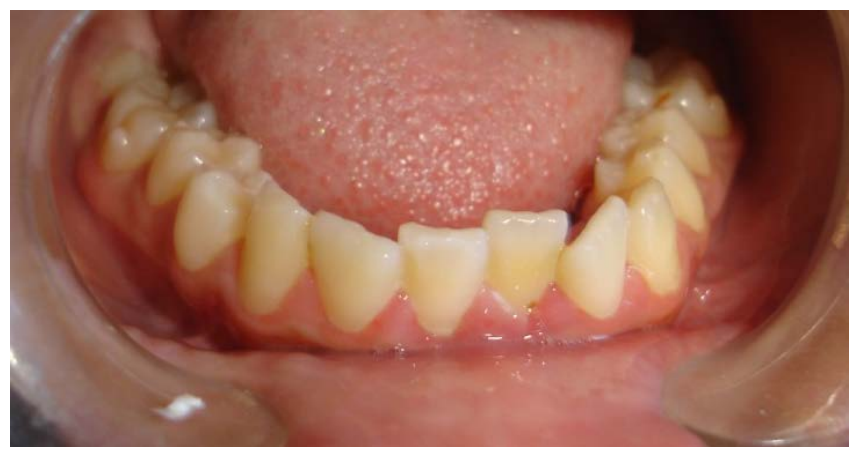

Figure 9. Frontal-intraoral view showing mild crowding in lower anterior teeth.

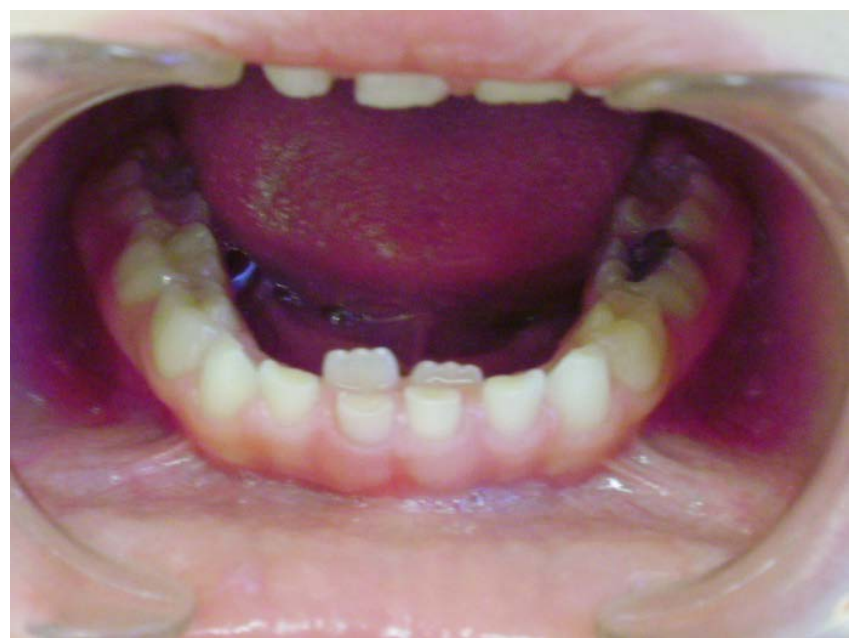

Figure 10. Frontal-intraoral view of lower teeth showing ectopic eruption of lower permanent incisors while primary incisors are retained.

Treatment of orthodontic discrepancy in the primary dentition may be indicated for correction of a posterior and/or anterior cross-bite, Class II or III malocclusions, premature loss of primary tooth, a cleft palate or crowding. Primary dentition treatment could begin at age of 4 to 5 [11]. This may be followed up with additional care in the early mixed dentition and more orthodontic treatment in the permanent dentition. The patient could potentially require three phases of orthodontic care from the ages of 4 to 15 . Another approach to early treatment is a two-phase approach. The first phase begins in the early mixed dentition at approximately age of 8 , and the second phase starts in the permanent dentition at approximately age of 12 . Some Orthodontists maintain that early mixed 


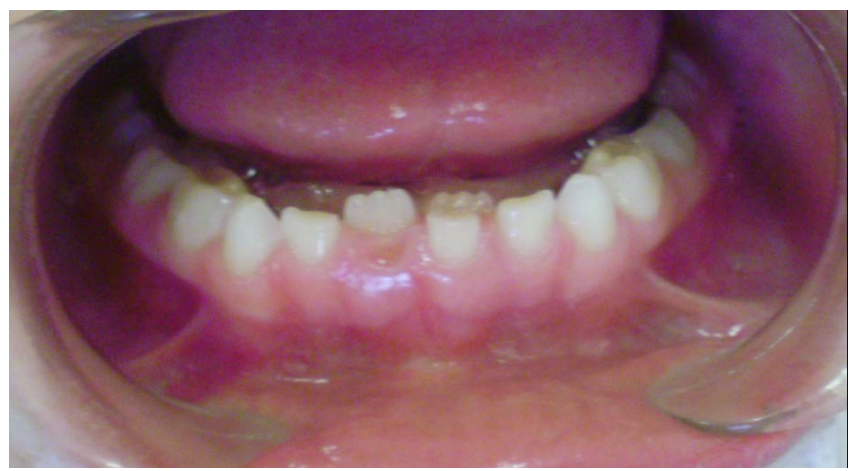

Figure 11. Frontal-intraoral view of lower teeth showing ectopic eruption of lower permanent incisors while one of primary incisors is exfoliated the other is still retained.

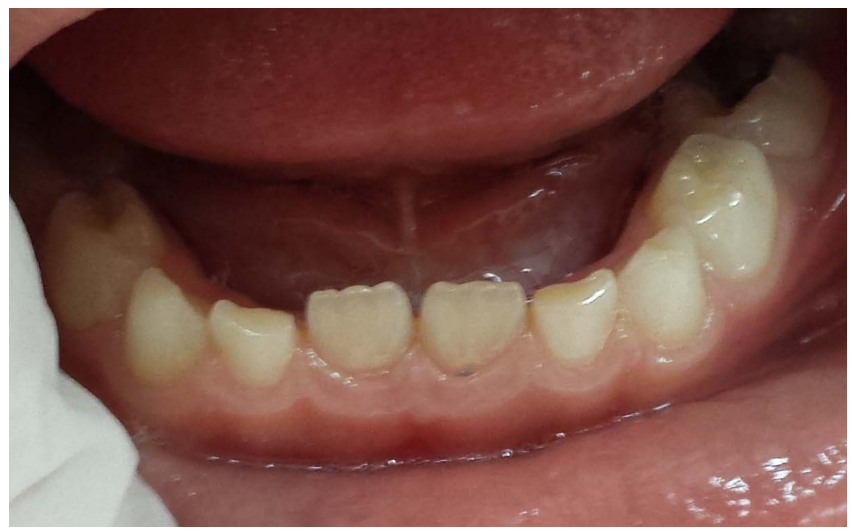

Figure 12. Frontal-intraoral view of lower teeth showing correction of ectopic eruption of lower permanent incisors after 3 months in comparison to Figure 10 and Figure 11.

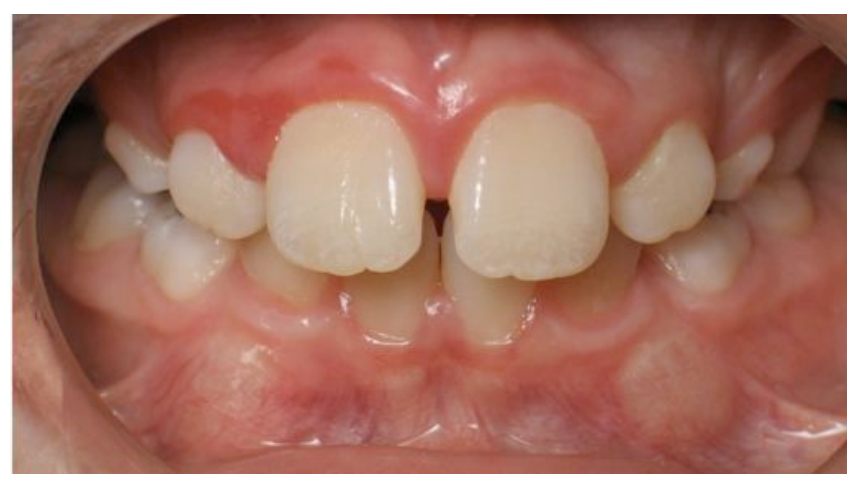

Figure 13. Frontal-intraoral view showing diastema between central Incisors in early ugly duckling stage.

dentition treatment with that Phase I orthodontic care can reduce or eliminate the need for full-banded Phase II orthodontic treatment at a laterage [10].

Others contend that Phase 1 treatment cannot produce lasting treatment results and that the patient will require a second phase of comprehensive care, which increases the number of office visits [11].

Orthodontic researchers have analyzed patients' compliance with early treatment and have shown different results. Some authors state that cooperation is better in the mixed dentition with younger patients than the older adolescent patients [10]. Others argue that early orthodontic treatment prolongs orthodontic care and the patient 


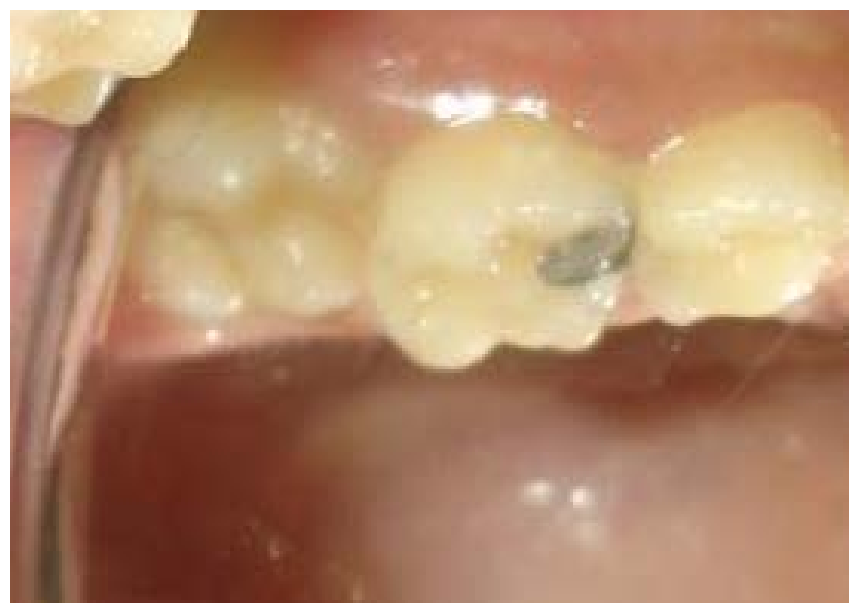

Figure 14. Lateral oblique-intraoral view showing ectopic eruption of maxillary first permanent molar.

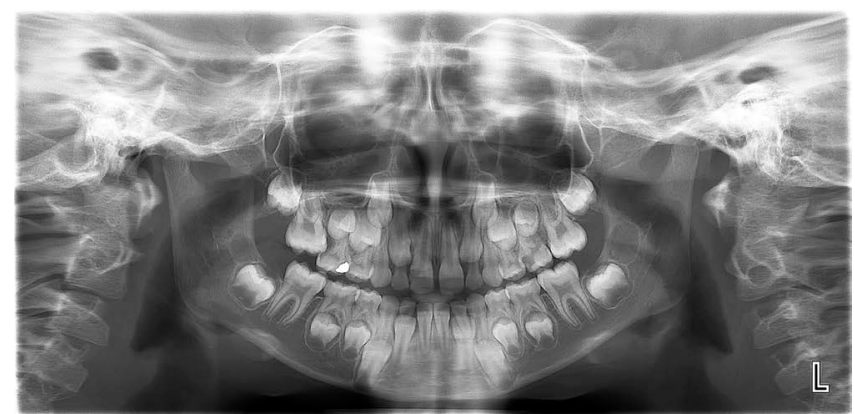

Figure 15. Orthopantogram showing Ectopic eruption of maxillary right first permanent molar.

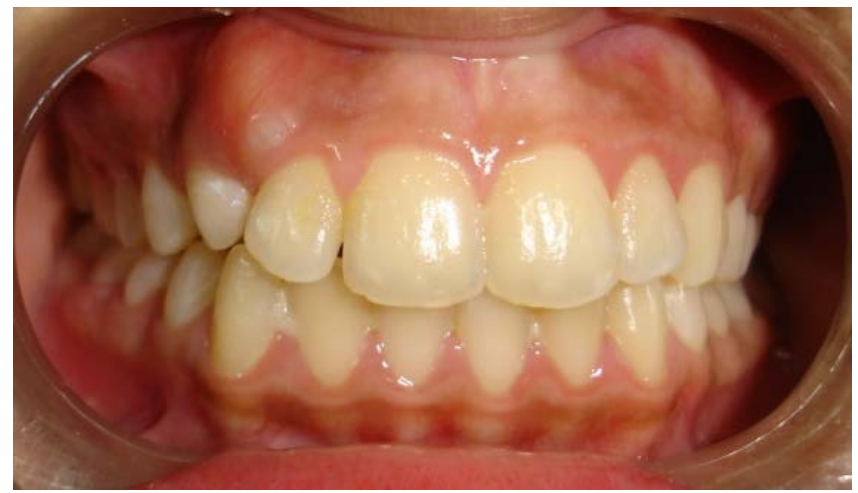

Figure 16. Frontal-intraoral view showing Ectopic eruption of maxillary right canine and the primary canine is still present.

will tend to "burn out" by the second phase of treatment [12]. Orthodontic treatment could begin in the late mixed dentition, at approximately age of 11 , and treatment would then be limited to one phase of orthodontic care. This approach can be effective in correcting many malocclusions; however, occasionally initiating orthodontic treatment in the late mixed dentition phase can extend treatment time as much as four years while waiting for eruption of all permanent teeth. Patients can experience "burn out" with this potential prolonged treatment time. Lastly, treatment could begin in the permanent dentition, which could shorten the treatment time and lessen the costs to the patient. The permanent dentition treatment would start upon eruption of the second molars, which may occur from the ages of 10 to 14 . Initiating treatment at this stage could present a problem with the physi- 


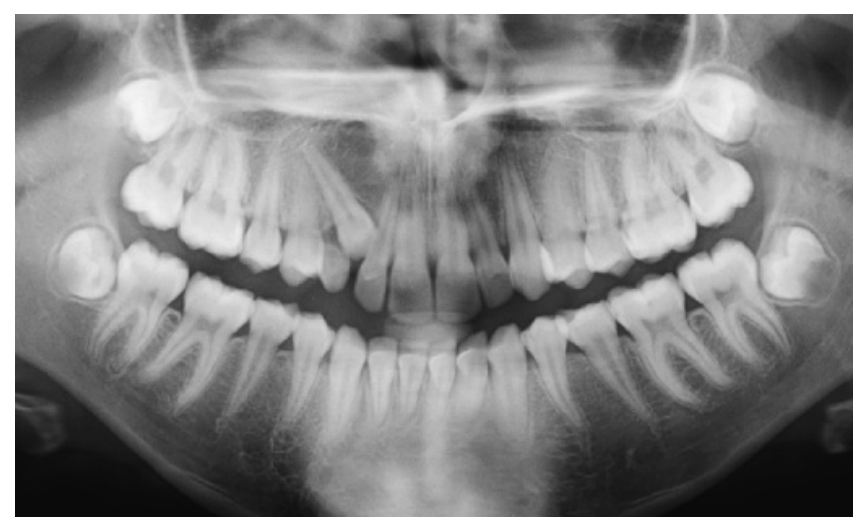

Figure 17. Orthopantogram showing ectopic eruption of maxillary right canine.

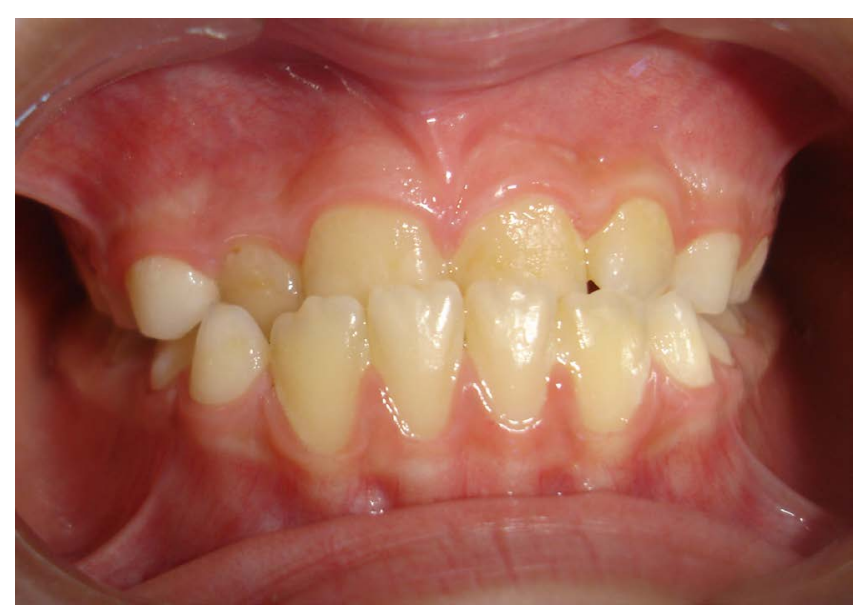

Figure 18. Frontal-intraoral view showing anterior cossbite as in pseudo Class III malocclusion.

cally mature female patient who might complete her growth before eruption of the second molars. If full-banded orthodontic care is initiated with little or no growth remaining, correcting the Class II malocclusion could become very difficult. There may be more needed for extractions, surgical orthodontics, or compromised orthodontic treatment when the patient has finished his or her facial growth. One reason for the controversy is that the accepted "cost" of an early treatment time is a two-phase protocol. Phase 1 generally involves 6 to 12 months of active treatment with the intent to change skeletodental relationships. Phase 2 is the "finishing" process after the eruption of appropriate permanent teeth. Thus, risk/reward analysis becomes inevitable. Do the benefits of early intervention justify the cost of two-phase treatment?

Two reports are of interest to define the scope of the problem. The Journal of Clinical Orthodontics survey of diagnosis and therapeutics [13] noted that approximately $25 \%$ of all patients were treated in a two-phase manner. The AAO May Bulletin indicated that approximately 1.3 million persons in 1992 elected orthodontic treatment. At $25 \%$ of penetration, at least $300,000+$ of patients are in a two-phase treatment program. There are only $900,000+$ of growing patients since adults comprise $20 \%$ to $25 \%$ of the patient population. Essentially, a third of all children are treated in two phases.

The premise is that at least $90 \%$ of all growing patients can be treated successfully in only one phase by starting treatment in the late mixed dentition stage of development-identified by the exfoliation of all deciduous teeth except the deciduous second molars or the "E”s. Implicit in this view is that there are few, if any, benefits that are unique to and dependent on earlier treatment. Also implicit is that habit control, the use of passive appliances such as space maintainers and minor alignment of incisors for esthetic or trauma reasons, is not considered part of conventional two-phase treatment. The other $5 \%$ to $10 \%$ of patients include those with crossbites 
complicated by a mandibular shift and certain patients with Class III malocclusions who could benefit from immediate resolution of the problem [13].

At the Department of Orthodontics, University of Pacific Arthur A. Dugoni School of Dentistry [14], a comprehensive mixed dentition treatment approach is taught to the orthodontic graduate students. This approach teaches the students to closely evaluate the entire malocclusion of patients who are approximately 7 to 8 years old. After thorough review of diagnostic records, a treatment plan is established to address the most or all of the problems present in the early mixed dentition. Treatment with the first phase is designed to correct all the problems. The goal is to eliminate or significantly reduce the need for Phase II orthodontic care. This approach helps to produce a less complicated problem in the second phase, shortening the overall treatment time.

The objectives of early treatment could include establishing ideal overjet and overbite, aligning the upper and lower incisors, establishing ideal torque, tip of the upper and lower incisors, adequate arch length, and obtaining a Class I molar position. Treatment typically uses fixed orthodontic appliances, including bands on the maxillary first molars and brackets on the upper incisors. Headgear would be used for correction of most Class II malocclusions. A facemask would be used to protract the maxilla forward in a Class III skeletal pattern. The mandibular arch is usually treated with a lingual arch that is removable and adjustable. If crowding is present in the maxillary and/or mandibular arch, the first primary molars or primary cuspids are extracted to gain room for alignment of the incisors. The mandibular lingual arch is adjusted at each visit until alignment of the incisors is obtained [14].

At the conclusion of the first phase of orthodontic treatment, the patient will enter a supervision stage until the eruption of permanent teeth. During this supervision stage, the patient wears a removable retainer and continues lingual arch to maintain the alignment of the lower incisors. Occasionally, headgear is worn during the supervision stage to continue correction of Class II molar position or to prevent rebound toward a Class II problem.

The investigators believe that the key to successful early treatment includes thorough and accurate diagnosis, comprehensive treatment planning, and continued care during supervision until the eruption of the permanent dentition. The best timing of orthodontic treatment is a decision made by the Orthodontist, the parent, and the patient based on all the factors that impact success. All options should be reviewed with the parent in order that he or she may make an informed decision. The American Association of Orthodontists, www.braces.org, recommends that all children get a check up with an orthodontist no later than age of 7 .

An early exam allows the orthodontist to offer advice and guidance as to when the optimal time to start treatment would be for that specific patient.

"The earlier treatment begins, the more the face will adapt to your standards; the later treatment begins, the more your standards will have to adapt to the face.”-C. Gugin. (Recent Concepts)—The 20th Century.

The 1997 Workshop Discussion on Early Treatment cautioned that "Iatrogenic problems may occur with early treatment such as dilaceration of roots, decalcification under bands left for too long, and impaction of maxillary second molars from distalizing first molars, and patient burnout [15].”

A recent study at the University of North Carolina concluded that "For children with moderate to severe Class II problems, early treatment followed by later comprehensive treatment does not produce major differences in jaw relationship or dental occlusion compared with later 1-stage treatment” [16]. Ferguson [17] wrote that 2-phase treatment was merely a means to capture patients for orthodontic treatment and prevented them from going elsewhere. Bowman [18] believed that today's trends are to treat earlier and often. He ridicules braces for baby teeth and asks, "Can in utero treatment be far off?” Turpin [19] summarized the results of the 2002 International Symposium on Early Orthodontic Treatment: Class II correction-A delay in treating Class II problems-Might not compromise treatment results, and it can increase efficiency. Patients with severe skeletal disharmony, excessive vertical development, and lack of cooperation obviously make it more difficult to achieve all objectives when treatment is limited to a single phase. A delay in starting treatment allows for self-correction of open bites in some patients, but this is unpredictable. Incisal contact is unlikely in most of these patients in the long term. Examine early to manage arch-length problems, including the need for disking deciduous teeth and selected extractions in some patients. Start treatment before the loss of the deciduous second molars, if possible. To correct Class III disharmony, diagnose and consider early treatment because of the unpredictability of growth. Don't expect total success in most patients in the long term. If disharmony is severe, delay treatment until you have proof that growth has ceased and included orthognathic surgery as a treatment option. Diagnose impaction and transposition and consider the need for early treatment because of the severity of complications that can be caused by unerupted and impacted teeth.” 
The best timing of orthodontic treatment is a decision made by the orthodontist, the parent, and the patient based on all the factors that impact success. All options should be reviewed with the parent in order that he or she may make an informed decision. An early exam allows the orthodontist to offer advice and guidance as to when the optimal time to start treatment would be for that specific patient [20].

\section{Conclusions}

1) The cornerstone of best management of orthodontic discrepancy is to identify its stability nature.

2) The best timing of orthodontic treatment is a decision made by the orthodontist, the parent, and the patient based on all the factors that impact success.

3) All options should be reviewed with the parent in order that he or she may make an informed decision.

4) An early exam allows the orthodontist to offer advice and guidance as to when the optimal time to start treatment would be for that specific patient.

\section{Acknowledgements}

First and foremost I would like to thank my Dad "Mohammad Naeem Mahfouz" Mom "Wedad Jarrad" Brother "Allam Mahfouz" Sisters "Haneen Mahfouz, Suha Mahfouz and Bayan Mahfouz "Wife "Yara Mansour" and Kids“Omar Mahfouz, Noor Mahfouz and Sara Mahfouz” for their valuable great support.

\section{References}

[1] Patti, A. and D’Arc, G.P. (2000) Clinical Success in Early Orthodontic Treatment. 1st Edition, Quintessence Publishing, New Malden, Surrey, 3-15.

[2] Dibiase, A. (2002) The Timing of Orthodontic Treatment. Dental Update, 29, 434-441.

[3] Proffit, W.R. and Ackerman, J.L. (1982) Diagnosis and Treatment Planning. In: Graber, T.M. and Swain, B.F., Eds., Current Orthodontic Concepts and Techniques, Chapter 1, Mosby, St. Louis, 3-100.

[4] Bailey, L.J., Proffit, W.R. and White Jr., R. (1999) Assessment of Patients for Orthognathic Surgery. Seminars in Orthodontics, 5, 209-222.

[5] Proffit, W.R. (2007) Contemporary Orthodontics. 4th Edition, Mosby, St. Louis, 107-129 and 689-691.

[6] Epker, B.N. and Wolford, L.M. (1980) Dentofacial Deformities: Surgical-Orthodontic Correction. Mosby, St. Louis.

[7] Katiyar, R., Singh, G.K., Mehrotra, D. and Singh, A. (2010) Ortho-Surgical Management of Skeletally Class III Malocclusion. National Journal of Maxillofacial Surgery, 1, 143-149. http://dx.doi.org/10.4103/0975-5950.79217

[8] Mahfouz, M. (2014) Pseudo Class III Treatment in 2-Year-Old Children. Open Journal of Stomatology, 4, 10-13. http://dx.doi.org/10.4236/ojst.2014.41003

[9] Mahfouz, M. (2014) Stainless Steel Double Loop Appliance to Correct Ectopically Positioned Maxillary Permanent First Molar. Open Journal of Stomatology, 4, 92-98. http://dx.doi.org/10.4236/ojst.2014.42015

[10] Dugoni, S., Lee, J., et al. (1995) Early Mixed Dentition Treatment: Postretention Evaluation of Stability and Relapse. The Angle Orthodontist, 65, 311-319.

[11] Tulloch, J.F., Proffit, W.R. and Phillips, C. (2004) Outcomes in a Two-Phase Randomized Clinical Trial of Early Class II Treatment. American Journal of Orthodontics and Dentofacial Orthopedics, 125, 657-667.

[12] Dugoni, S.A. (1998) Comprehensive Mixed Dentition Treatment. American Journal of Orthodontics \& Dentofacial Orthopedics, 113, 75-84. http://dx.doi.org/10.1016/S0889-5406(98)70278-1

[13] Gianelly, A.A. (1995) One-Phase versus Two-Phase Treatment. American Journal of Orthodontics \& Dentofacial Orthopedics, 108, 556-559. http://dx.doi.org/10.1016/S0889-5406(95)70057-9

[14] Dugoni, S.A. and Aubert, M.M. (2006) Orthodontic Treatment in the Early Mixed Dentition: Is This the Optimum Time to Start Care? Journal of the California Dental Association, 10, 807-812.

[15] Wahl, N. (2006) Orthodontics in 3 Millennia. Chapter 12: Two Controversies: Early Treatment and Occlusion. American Journal of Orthodontics \& Dentofacial Orthopedics, 130, 799-804.

[16] Tulloch, J.F.C., Phillips, C. and Proffit, W.R. (1998) Benefit of Early Class II Treatment: Progress Report of a TwoPhase Randomized Clinical Trial. American Journal of Orthodontics \& Dentofacial Orthopedics, 113, 62-72. http://dx.doi.org/10.1016/S0889-5406(98)70277-X 
[17] Ferguson, J. (1996) Comment on Two-Phase Treatment. American Journal of Orthodontics \& Dentofacial Orthopedics, 110, 14A-15A.

[18] Bowman, J. (1998) One-Stage versus Two-Stage Treatment: Are Two Really Necessary? American Journal of Orthodontics \& Dentofacial Orthopedics, 113, 111-116. http://dx.doi.org/10.1016/S0889-5406(98)70283-5

[19] Turpin, D.L. (2002) Early Treatment Conference Alters Clinical Focus. American Journal of Orthodontics \& Dentofacial Orthopedics, 121, 335-336. http://dx.doi.org/10.1067/mod.2002.123783

[20] Grover, N., Verma, S. and Dhinsa, K. (2013) Early versus Late Orthodontic Treatment. Lambert Academic Publishing, AG \& Co.KG. 